\title{
Machine learning paves the way to advances in genome sequencing
}

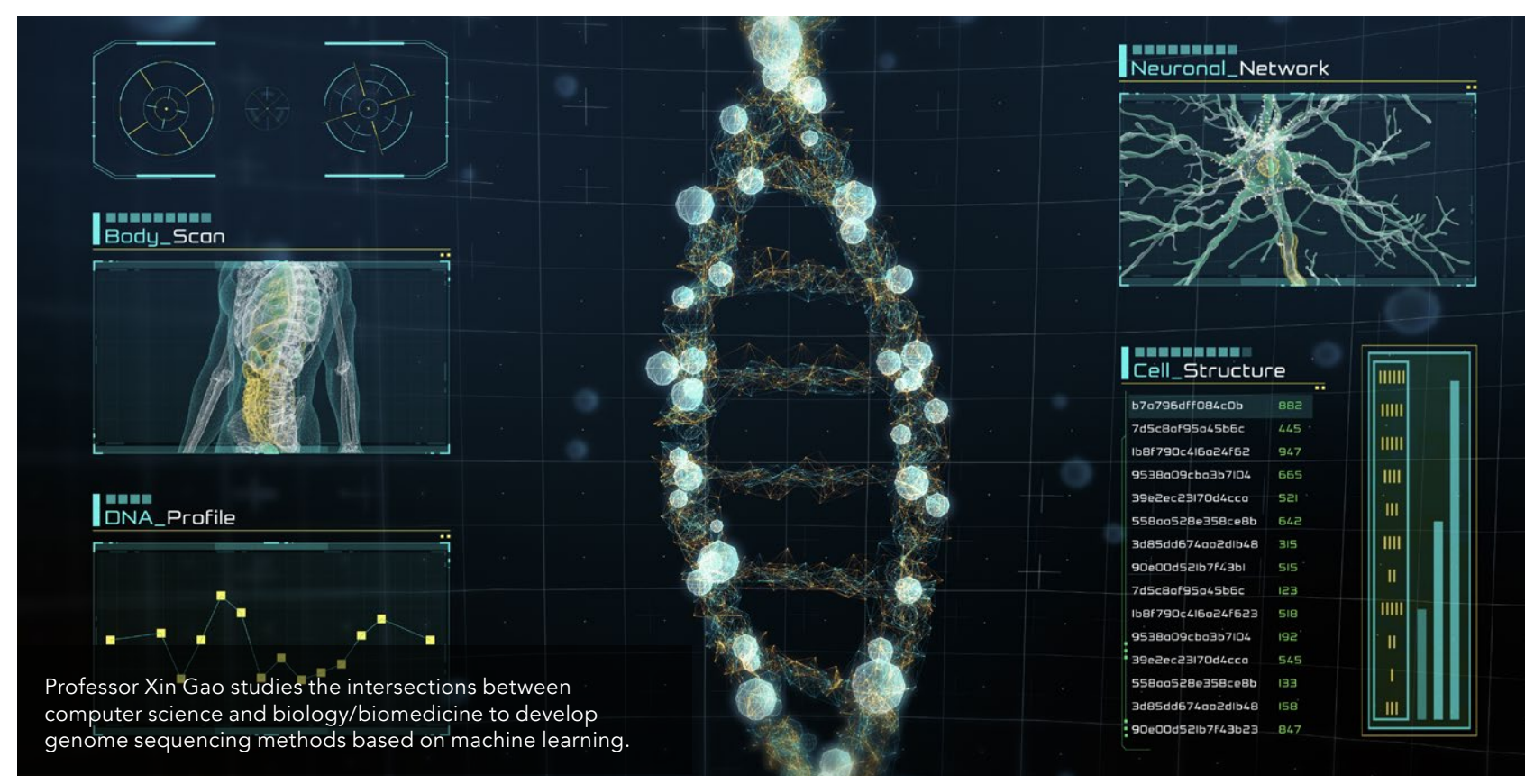

Genome sequencing platforms are transforming the field of genetic disease research as they offer a closer look at human genes and Deepsimulator provides simulated datasets to train and test sequencing DeepSimulator provides simulated datasets to train and test sequencing analyicit ol w r w professor of computer science in CEMSE Division at King Abdullah University of Science and Technology (KAUST), Associate Director of Computational Bioscience Research Center, and Deputy Director of Smart Health Initiative at KAUST. With his team he studies the intersections between computer science and biology/biomedicine to develop genome sequencing methods based on machine learning.

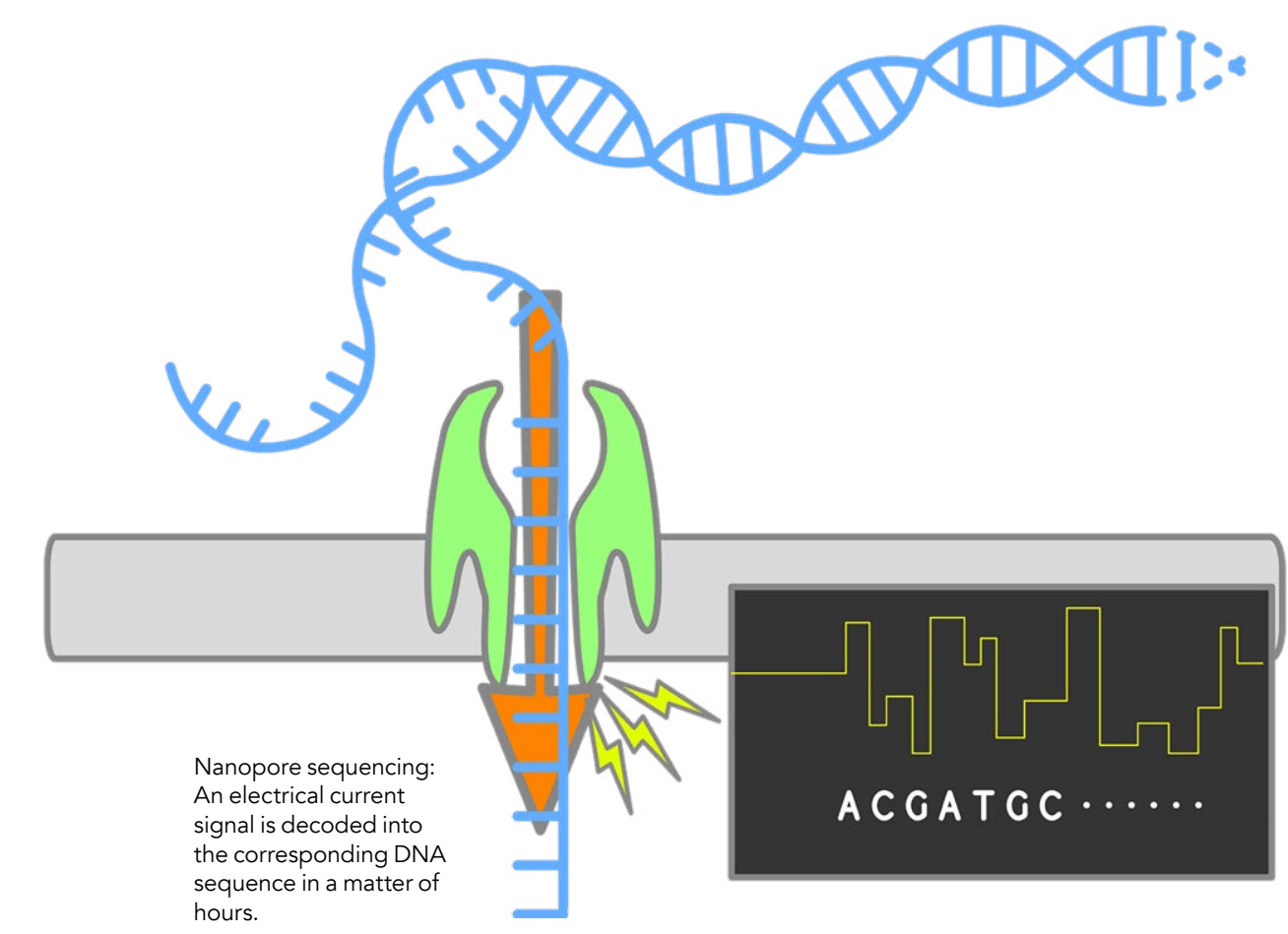

ome diseases such as cystic occur as a result of an error in 'single-gene disorders'. These so-called Mendelian Disorders are passed down through generations which presents an opportunity to researchers: if they can fix the gene error, they will eliminate the disease.

Genome sequencing is the process of determining a DNA sequence of an organism's genes usually with the purpose of identifying mutations, prestecade, DNA. Over the past decade, he technology has diseases such as the Mendelian Disorders as researchers are able detect genetic changes in a sample with great precision.

A NOVEL SEQUENCING TOOL One of these genome sequencing tools is the Nanopore third generation sequencing platform, the MinlON, which is a portable device the size of an iPhone. Wthout the need for PCR amplification, the MinION is simple to use and particularly good at reading Min loN stretches of DNA. The wells made of proteins, or nanopores, with an electrical current running through the system. The nanopores are small enough to fit a single strand of
nucleic acids (DNA or RNA) disrupting the current as it passes through the as base-calling, takes the electrical current signal and outputs a segmentec curve which is then decoded into the corresponding DNA sequence in a matter of hours.

Despite the MinlON offering genome sequencing both fast and accessibly, certain DNA errors, so-called indels, are frequently not identified in the final sequence output. This is where the research of Professor Gao and his team at The Structural and Functiona Bionformatics group, King Abdullah University of Science and Technology how this shorconing is the divided base-calling process and present a novel base-alling method WaveNano, borrowing techniques

from speech recognition deep machine learning (a type of artificial intelligence able to learn from large datasets without human interference). By considering the nanopore signal as a speech signal, the base-calling process can then effectively be viewed as speech recognition. WaveNano jumps over the traditional segmentation step and directly decodes the raw signal sequence substantially reducing the amount of indel errors.

SIMULATED VALIDATION The large amount of output data then needs to be analysed with the DNA sequence from the MinION reco a reference genome in order to Due to the rapid growth of the field, validating the efficiency of new analytical tools is not always possible due to lack of empirical data (e.g. annotated data or patient samples), Prof Gao and his team have developed the first signal-level simulator for Nanopore: DeepSimulator, which imitates the entire physical process of Nanopore sequencing using de researcher is able to generate sample

\section{DeepSimulator provides simulated} datasets to train and test sequencing analytical tools, cwDTW provides a highly efficient solution to align raw signals with DNA reads, while WaveNano has innovated the process of translating a sample into a DNA sequence.

datasets to test and validate analytical tools from a known starting sequence, or ground truth. Starting from a given reder creates simulated electrical cur signals by a context-dependent methods for Nanopore sequencing data analysis, Deepsimulator also helps researchers better understand empirical datasets. Due to the high using DepSimutar and requests on customizing the 


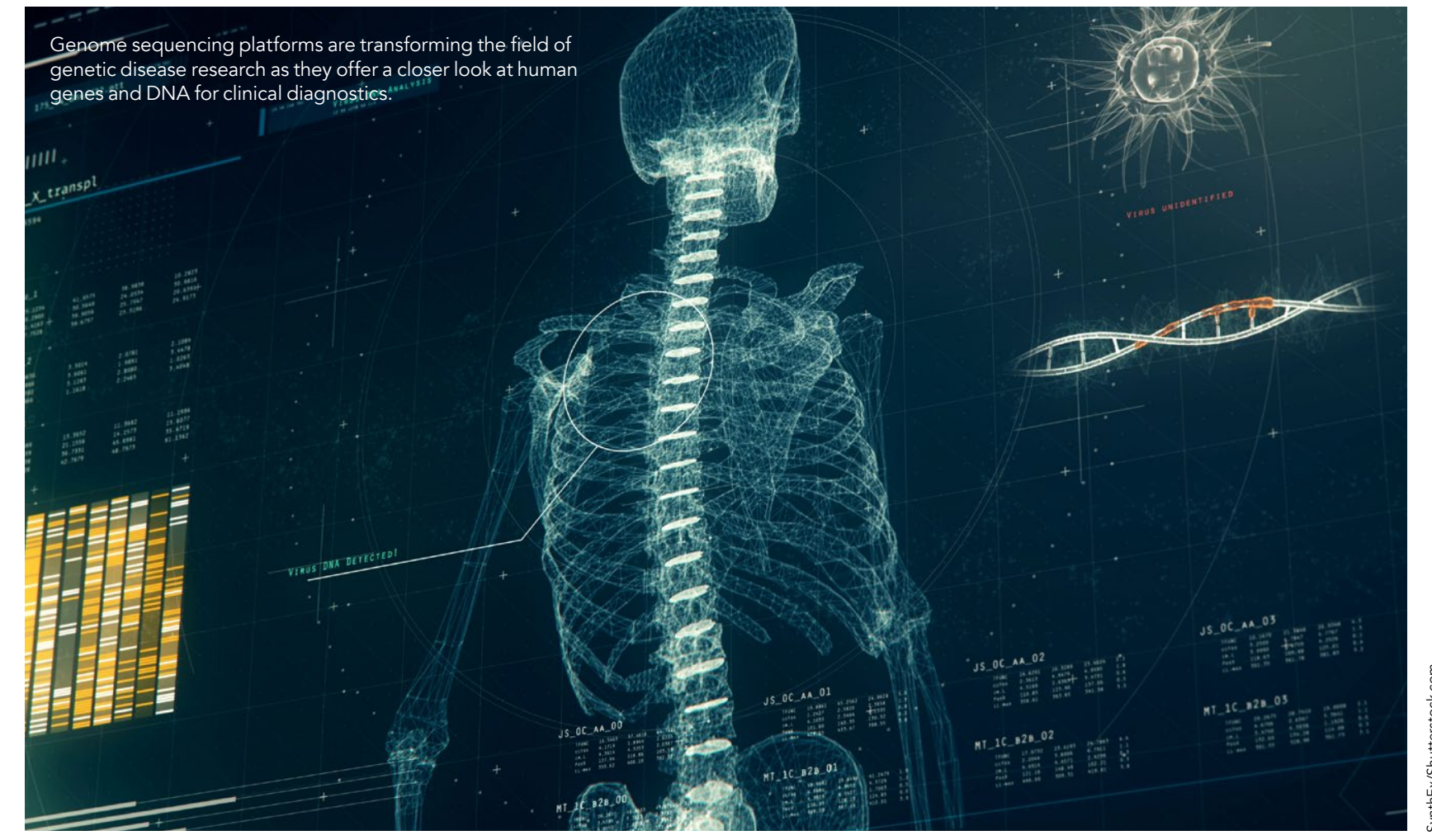

simulator, Prof Gao recently developed DeepSimulator 1.5 .

of researchers identified large structural variations induced by the unique gene editing tool CRISPR-Cas9 in human stem cells. This could both help the safe editing technolovement of the gene (a) variations in patients. Additionally. Prof the raw signal sequences with the DNA reads, both of which are extremely long: the DNA reads of Nanopore range

between $10 \mathrm{~K}$ to $100 \mathrm{~K}$, whereas the raw signal sequence is even ten times longe. Gao's team was able to diagnose gen Nanopore sequencer and the To this end, they developed a highly
efficient alignment efficient alignment
algorithm, cwDTW, algorithm, CWDTW,
which is 3000 times faster than the original dynamic time warping (DTW) algorithm Prof Gao's team diagnosed genetic RNA Saudi Arabia and variants in $\mathbf{1 3 . 5 \%}$ more patients who had 25 start-ups among previously been declared 'negative' by in alignment.

Pushing the methods further into applications in various scientific domains, Prof Gao tested the in-house end-toend pipeline on clinical diagnosis of genetic diseases, antibiotic resistance gene detection, and the effect of genomic editing. In some cases, rare genetic variants are missed by the genome sequencing systems which has devastating effects on patients if mutations are not discovered in time. By labelling and sequencing individual
RNA variants in $13.5 \%$ more patients who had previously been declared 'negative' so, the study paved the way to combine the raw signal of patient samples from sequencing and analysis.

\section{GENOMIC DIAGNOSTICS}

'ANYWHERE, ANYTIME'

Through their academic collaboration Professors Gao and Mo Li co-founded the start-up, Peregrine Genomics, with a goal of providing real-time geno diagnostics 'anywhere, anytime'.

Biomedical Genomics Anywhere Anyti he sequencer, the machine learning real-time.

During the past three years, Prof $G a o$ and his team have developed a series of methods and algorithm to provide an end-to-end pipeline for Nanthing from becic re covering ratical and linicac applications, and clinical RNA diagnostics with genome

\section{Behind the Research}

\section{Professor Xin Gao}

E: xin.gao@kaust.edu.sa T: +966-12-8080323 W: $\underline{\text { http://sfb.kaust.edu.sa }}$

The Paregrine Genomics system combines the portable long-reads innovative data analysis solution, in the hope of overcoming the curren technical imperfection in genetic diagnosis and accelerate its application to human healthcare. In September 2019, the start-up won the Taqadam ups from all over
the world at the Entrepreneurship

\section{Research Objectives}

Professor Gao's research interest lies at the intersection between computer science and biology. In the field machine learning theories and methodologies related to deep learning, probabilistic graphical models, kernel methods and matrix factorisation. In the field of bioinformatics, his group works on building techniques, and designing efficient and effective lgorithms to tackle key open problems along the path from biological sequence analysis, to 3D structure determination, function annotation, understanding and controlling molecular behaviours in complex biological

\section{Detail}

Xin Gao
Building 3, Room 4217, CBRC, KAUST, Thuwal, 23955, Saudi Arabia

WaO is Professor of computer science in CEMSE Divisio CAUST. He is also the Acting Associate Director of the Computational Bioscience Research Center (CBRC), Deputy Director of the Smart Health Initiative (SH), and the lead of Prior to jual and KAUSTional Bioinformatics Group at KAUST. for Computational Biology in School of Computer Science at Carnegie Mellon University. He earned his BSc in Computer Science, Tsinghua University in 2004 and PhD in Computer Science, University of Waterloo, 2009. He has published more than 230 papers in the fields of bioinformatics and machine learning. He is associate editor of Genomics,
Proteomics \& Bioinformatics, BMC Bioinformatics, Journal of Bioinformatics and Computational Biology, and Ountitative Biology, and the guest editor-in-chief of IEEE/ACM Transactions on Computational Biology and Bioinformatics, Methods, and Frontiers in Molecular Bioscience.

Funding
Collaborators

Professor Mo Li, BESE Division at KAUS

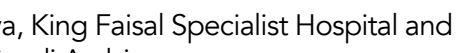
Research Center Saudi Arabia

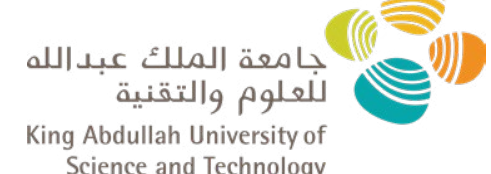

\section{References}

S. Wang, Z. Li, Y. Yu, and X. Gao. (2018). WaveNano: a signal-level nanopore base-caller via simultaneous prediction of nucleotide labels and move labels through bidirectional WaveNets. Quantitative Biology. 6(4): 359-368.

Y. Li, R. Han, C. Bi, M. Li, S. Wang, and X. Gao. (2018). Deepsimulator: a deep simulator for Nanopore sequencing

Y. Li, S. Wang, C. Bi, Z. Qiu, M. Li, and X. Gao. (2020). DeepSimulator1.5: a more powerful, quicker and lighter DeepSimulator. S: a more powerful, quicker and lighter 2578-2580.

R. Han, Y. Li, X. Gao, and S. Wang. (2018). An accurate and rapid continuous wavelet dynamic time warping algorithm for end-to-end mapping in ultra-long nanopore sequencing. Bioinformatics. 34(17): i722-i731.

R. Han, S. Wang, and X. Gao. (2019). Novel algorithms for efficient subsequence searching and mapping in nanopore raw signals towards targeted sequencing. Bioinformatics. 36(5): 1333-1343.

S. Maddirevula, et al. (2020). Analysis of transcriptRNA-based diagnostics. Genome Biology. 21: 145.

C. Bi, L. Wang, B. Yuan, X. Zhou, Y. Li, S. Wang, Y. Pang, X. Gao, Y. Huang, and M. Li. (2020). Long-read individualX heterogeneity in human ESCs. Genome Biology. 21: 213.

\section{Personal Response}

Based on your research, what do you think is the next step in genome sequencing?

II In my opinion, the mutations, insertions and

deletions have been relatively well solved in genome a big challenge. Therefore, lexpect the next step is to develop computational methods to resolve SVs. In them with traits or diseases is another key challenge in the field. 